\title{
A comparative study between data obtained from conventional lateral cephalometry and reconstructed three-dimensional computed tomography images
}

\author{
Suseok $\mathrm{Oh}^{1}$, Ci-Young Kim², Jongrak Hong ${ }^{1}$ \\ ${ }^{1}$ Department of Oral and Maxillofacial Surgery, Samsung Medical Center, Sungkyunkwan University School of Medicine, \\ ${ }^{2}$ Department of Nursing, Hanyang University College of Medicine, Seoul, Korea
}

\begin{abstract}
J Korean Assoc Oral Maxillofac Surg 2014;40:123-129)
Objectives: The aim of this study was to verify the concordance of the measurement values when the same cephalometric analysis method was used for two-dimensional (2D) cephalometric radiography and three-dimensional computed tomography (3D CT), and to identify which 3D Frankfort horizontal (FH) plane was the most concordant with FH plane used for cephalometric radiography.

Materials and Methods: Reference horizontal plane was FH plane. Palatal angle and occlusal plane angle was evaluated with FH plane. Gonial angle (GA), palatal angle, upper occlusal plane angle (UOPA), mandibular plane angle (MPA), U1 to occlusal plane angle, U1 to FH plane angle, SNA and SNB were obtained on 2D cephalmetries and reconstructed 3D CT. The values measured eight angles in 2D lateral cephalometry and reconstructed 3D CT were evaluated by intraclass correlation coefficiency (ICC). It also was evaluated to identify 3D FH plane with high degree of concordance to 2D one by studying which one in four FH planes shows the highest degree of concordance with 2D FH plane.

Results: ICCs of MPA (0.752), UOPA (0.745), SNA (0.798) and SNB (0.869) were high. On the other hand, ICCs of gonial angle (0.583), palatal angle (0.287), U1 to occlusal plane (0.404), U1 to FH plane (0.617) were low respectively. Additionally GA and MPA acquired from 2D were bigger than those on 3D in all 20 patients included in this study. Concordance between one UOPA from 2D and four UOPAs from 3D CT were evaluated by ICC values. Results showed no significant difference among four FH planes defined on 3D CT.

Conclusion: FH plane that can be set on 3D CT does not have difference in concordance from FH plane on lateral cephalometry. However, it is desirable to define FH plane on 3D CT with two orbitales and one porion considering the reproduction of orbitale itself.
\end{abstract}

Key words: Lateral cephalography, Three-dimensional computed tomography, Frankfort horizontal plane

[paper submitted 2014. 5. 16 / revised 2014. 6. 12 / accepted 2014. 6. 13]

\section{Introduction}

Accurate diagnosis is the most critical assignment in planning orthognathic surgery, and conventional lateral cephalometry has been the standard for evaluation and orthodontic diagnosis of maxillofacial deformity since the early $1930 \mathrm{~s}^{1}$.

\section{Jongrak Hong}

Department of Oral and Maxillofacial Surgery, Samsung Medical Center, Sungkyunkwan University School of Medicine, 81 Irwon-ro, Gangnam-gu, Seoul 135-710, Korea

TEL: +82-2-3410-2420 FAX: +82-2-3410-0038

E-mail: hongjr@skku.edu

(c) This is an open-access article distributed under the terms of the Creative Commons Attribution Non-Commercial License (http://creativecommons.org/licenses/by-nc/3.0/), which permits unrestricted non-commercial use, distribution, and reproduction in any medium, provided the original work is properly cited.

Copyright (C) 2014 The Korean Association of Oral and Maxillofacial Surgeons. All rights reserved.
Various methodologies have been introduced, and normal average value (norm) for corresponding analysis has been reported $^{2}$. Analysis of lateral cephalometry includes the analysis of angle and length of hard tissues and soft tissues of the face and is the most commonly used method ${ }^{3}$. However, twodimensional (2D) lateral cephalometry is associated with many problems. First, it is difficult to achieve accurate identification due to landmark overlap ${ }^{4-6}$. Second, it is difficult to clearly reflect the difference between the right and left sides of the face. Third, there is a fundamental problem in that a threedimensional (3D) subject is illustrated in two dimensions?

In addition, weaknesses of 2D lateral cephalometry have been reported including low concordance with results of actual clinical evaluation ${ }^{8,9}$. Conventional lateral cephalometry has another limitation in that deficiency in the paranasal and

This research was supported by a grant of the Korea Health Technology R\&D Project through the Korea Health Industry Development Institute funded by the Ministry of Health \& Welfare, Republic of Korea (HI13C1491). 
infraorbital areas cannot be accurately demonstrated.

$3 \mathrm{D}$ facial analysis has been presented to solve such shortcomings and can be used to achieve simulation treatment for $3 \mathrm{D}$ operation and orthodontic processes ${ }^{10-13}$. Systems have been established for normal average values, landmarks, reference lines, and reference planes, all of which can serve as standards of analysis ${ }^{14}$. If a landmark is defined for 3D analysis, such landmarks should be used for 2D analysis in most cases. This is because lateral cephalometry analysis has been widely performed, and the massive amount of data accumulated can be effectively used for 3D analysis ${ }^{15}$. However, few studies have analyzed whether 3D analysis agrees with the large amount of $2 \mathrm{D}$ data.

In this regard, this study analyzed the concordance and patterns between eight angles measured using lateral cephalometry as well as 3D computed tomography (CT) images. In addition, it also aims to identify a 3D Frankfort horizontal (FH) plane with a high degree of concordance to the $2 \mathrm{D}$ plane by studying four FH planes.

\section{Materials and Methods}

\section{Patient selection and data collection}

The study population consisted of 20 patients who visited the Department of Oral and Maxillofacial Surgery, Samsung Medical Center (Seoul, Korea) in 2012 who underwent lateral cephalometry (Planmeca ProMax; Planmeca Oy, Helsinki, Finland) and 3D CT (GE LightSpeed VCT XT; General Electronic Medical System, Milwaukee, WI, USA) imaging. All patients were skeletally mature and no longer growing. In total, 20 patients were selected among whom there were 12 males and eight females with an average age of 23.2 years.

The area from the nasal bone to the mandible was included in the image, and the axial slice thickness was $1 \mathrm{~mm}$. During CT scanning, the jaw and teeth were maintained in centric occlusion. Facial bone CT was taken with the patient in a supine position. After completion of CT scanning, the images were saved as digital imaging and communication in medicine (DICOM) files. CT images were also reconstructed to produce 3D images using Simplant Pro 2011 (Materialize Dental NV, Leuven, Belgium).

The following patients were excluded: those with severe congenital malformation, such as hemifacial microsomia, those with pathological lesions of the jaw, and those with severe inflammation.

In the reconstructed 3D image, lines, planes, and angles were set as follows, and the FH plane was the horizontal reference plane in this study.

Lateral cephalometry images were analyzed using V-ceph

Table 1. Definitions of the angles, linear measurements, and planes

\begin{tabular}{|c|c|c|}
\hline \multirow{2}{*}{ Measurements } & \multicolumn{2}{|c|}{ Definition } \\
\hline & 2D cephalometry & Reconstructed 3D CT \\
\hline \multicolumn{3}{|l|}{ Angular measurement } \\
\hline Gonial angle & Angle from $\mathrm{Me}$ to the Go to the $\mathrm{Ar}$ & Average between right and left gonial angles \\
\hline Palatal angle & Angle between palatal line and $\mathrm{FH}$ line & Same as 2D cephalometry \\
\hline Mandibular plane angle & Angle between SN line and Mn plane line & Same as $2 \mathrm{D}$ cephalometry \\
\hline Upper occlusal plane angle & Angle between upper occlusal line and FH plane & Angles between upper occlusal line and each FH plane \\
\hline U1 to occlusal plane angle & Angle between U1 line and upper occlusal line & Same as $2 \mathrm{D}$ cephalometry \\
\hline U1 to FH plane angle & Angle between U1 line and FH plane & Angle between U1 line and each FH plane \\
\hline SNA & Angle from sella to the nasion to the subspinal point & Same as $2 \mathrm{D}$ cephalometry \\
\hline SNB & Angle from sella to the nasion to the supramental point & Same as $2 \mathrm{D}$ cephalometry \\
\hline \multicolumn{3}{|l|}{ Linear measurement } \\
\hline Upper occlusal line & Line through tip of central incisor and MB cusp of first molar & $\begin{array}{l}\text { Line through (midpoint between \#16 and \#26) and } \\
\text { (midpoint between central incisors tip) }\end{array}$ \\
\hline Mandibular plane line & Line through corpus left and $\mathrm{Me}$ & Line through (midpoint between Go) and Me \\
\hline Palatal line & Line through ANS and PNS & Same as 2D cephalometry \\
\hline U1 line & Line through tip and apex of central incisor & Line through tip and apex of central incisor \\
\hline \multicolumn{3}{|l|}{ Plane measurement } \\
\hline FH plane & Line through Or and Po & - \\
\hline FH plane 1 & - & Plane through both Or and right Po \\
\hline FH plane 2 & - & Plane through both Or and left Po \\
\hline FH plane 3 & - & Plane through right Or and both Po \\
\hline FH plane 4 & - & Plane through left Or and both Po \\
\hline
\end{tabular}

(2D: two-dimensional, 3D: three-dimensional, Me: menton, Go: gonion, Ar: articulare, FH: Frankfort horizontal, SN: sella nasion, Mn: mandible, U1: upper 1st incisor, MB: mesiobuccal, Or: orbitale, Po: porion)

Suseok Oh et al: A comparative study between data obtained from conventional lateral cephalometry and reconstructed three-dimensional computed tomography images. J Korean Assoc Oral Maxillofac Surg 2014 
5.5 (CyberMed Inc., Seoul, Korea). All analysis was performed by a single investigator.

\section{Concordance evaluation between the $\mathrm{FH}$ planes} defined on $2 \mathrm{D}$ and $3 \mathrm{D}$ systems

The FH plane on lateral cephalometry was defined with the lines passing the orbitale (Or) and porion (Po), and the upper occlusal plane angle (UOPA) was measured. Four FH planes (Table 1) were established in the 3D system, and the UOPA of each point was measured. The angles measured in 3D and lateral cephalometry were evaluated with regard to intraclass correlation coefficient (ICC) with IBM SPSS Statistics 21.0 (IBM Co., Armonk, NY, USA).

\section{Concordance evaluation between measured angles} from 2D and $3 \mathrm{D}$ systems

(1) Gonial angle (GA), (2) palatal angle, (3) mandibular plane angle (MPA), (4) UOPA, (5) U1 to occlusal plane angle (U1OPA), (6) U1 to FH plane angle (U1FHPA), (7) SNA, (8) SNB were measured both in lateral cephalometry and reconstructed 3D CT (Table 1) and were evaluated with regard to ICC.

\section{Results}

1. Concordance evaluation between $\mathrm{FH}$ planes defined on 2D and 3D systems

On lateral cephalometry, the average UOPA was $11.38^{\circ}$. When the concordance of UOPA against the four FH planes on 3D was evaluated, the ICCs showed high concordance with $0.745,0.751,0.755$, and 0.754 , respectively. The results

Table 2. Average UOPA values on $2 \mathrm{D}$ cephalometry and $3 \mathrm{D}$ CT and analysis of reliability $\left({ }^{\circ}\right)$

\begin{tabular}{lccc}
\hline & 2D cephalometry & Reconstructed 3D CT & ICC \\
\hline UOPA & 11.38 & - & - \\
UOPA 1 & - & 10.83 & $0.745^{*}$ \\
UOPA 2 & - & 11.82 & $0.751^{*}$ \\
UOPA 3 & - & 11.07 & $0.755^{*}$ \\
UOPA 4 & - & 11.66 & $0.754^{*}$ \\
\hline
\end{tabular}

(UOPA: upper occlusal plane angle, 2D: two-dimensional, 3D CT: three-dimensional computed tomography, ICC: intraclass correlation coefficient)

*Statistical significance $(P<0.05)$.

Suseok Oh et al: A comparative study between data obtained from conventional lateral cephalometry and reconstructed three-dimensional computed tomography images. J Korean Assoc Oral Maxillofac Surg 2014 were not significantly different. The ICC differences among the four angles acquired in $3 \mathrm{D}$ were also not significant different.(Table 2)

\section{Concordance evaluation between measured angles from 2D and 3D systems}

Concordance of the average of eight angles was verified. (Table 3) ICCs of GA, palatal angle, U1OPA, and U1FHPA were low at $0.583,0.287,0.404$, and 0.617 , respectively. Thus demonstrating a low concordance between lateral cephalometry and 3D CT. On the other hand, ICCs of MPA, UOPA, SNA, and SNB were high at 0.752, 0.745, 0.798, and 0.869 , respectively. Additionally, GA and MPA acquired in 2D were larger than those in $3 \mathrm{D}$ for all 20 patients included in this study.(Table 4)

\section{Discussion}

Two-dimensional cephalometry has been widely used for diagnosis and operation in craniofacial skeletal development and orthodontic and orthognathic surgeries ${ }^{13,16}$. This analysis has been used to analyze lines and angles on an image. The measured values from the patients can be compared with generally known normal values to judge the asymmetry ${ }^{17}$. In lateral cephalometry, however, it is difficult to distinguish the overlapping structures; in patients with an asymmetric face, the accuracy of analysis is low. Consequently, it is desirable to also use other radiographic images in order to achieve more accurate analysis.

To evaluate the asymmetry of a face, panorama radiography ${ }^{18}$ and posteroanterior cephalometry have been tradition-

Table 3. Average correlations between two groups $\left({ }^{\circ}\right)$

\begin{tabular}{lccc}
\hline & 2D cephalometry & Reconstructed 3D CT & ICC \\
\hline GA & 126.80 & 120.24 & 0.583 \\
Palatal angle & 2.00 & 0.05 & 0.287 \\
U1OPA & 52.16 & 52.91 & 0.404 \\
U1FHA & 116.47 & 116.56 & 0.617 \\
MPA & 38.04 & 33.56 & 0.752 \\
UOPA & 11.38 & 10.83 & 0.745 \\
SNA & 84.82 & 84.22 & 0.798 \\
SNB & 83.21 & 83.64 & 0.869 \\
\hline
\end{tabular}

(2D: two-dimensional, 3D CT: three-dimensional computed tomography, ICC: intraclass correlation coefficient, GA: gonial angle, U1OPA: U1 to occlusal plane angle, U1FHA: U1 to FH plane angle, MPA: mandibular plane angle, UOPA: upper occlusal plane angle) Suseok Oh et al: A comparative study between data obtained from conventional lateral cephalometry and reconstructed three-dimensional computed tomography images. J Korean Assoc Oral Maxillofac Surg 2014 
Table 4. GA and MPA obtained from all patients

\begin{tabular}{|c|c|c|c|c|}
\hline \multirow{2}{*}{ Patient No. } & \multicolumn{2}{|c|}{$\mathrm{GA}\left({ }^{\circ}\right)$} & \multicolumn{2}{|c|}{$\operatorname{MPA}\left({ }^{\circ}\right)$} \\
\hline & 2D cephalometry & Reconstructed 3D CT & 2D cephalometry & Reconstructed 3D CT \\
\hline 1 & 121.21 & 117.13 & 38.44 & 35.21 \\
\hline 2 & 120.91 & 114.89 & 30.31 & 26.93 \\
\hline 3 & 128.54 & 122.70 & 42.43 & 38.13 \\
\hline 4 & 125.29 & 122.16 & 33.86 & 28.27 \\
\hline 5 & 118.96 & 115.70 & 37.73 & 32.56 \\
\hline 6 & 135.06 & 128.10 & 40.83 & 37.52 \\
\hline 7 & 126.54 & 122.09 & 42.97 & 37.24 \\
\hline 8 & 127.99 & 118.90 & 45.44 & 39.06 \\
\hline 9 & 115.46 & 109.77 & 29.54 & 25.26 \\
\hline 10 & 138.15 & 130.11 & 38.05 & 30.72 \\
\hline 11 & 137.88 & 127.64 & 36.31 & 35.97 \\
\hline 12 & 124.88 & 122.86 & 36.01 & 31.75 \\
\hline 13 & 109.51 & 104.14 & 22.86 & 21.30 \\
\hline 14 & 133.72 & 125.29 & 46.00 & 38.52 \\
\hline 15 & 127.27 & 126.17 & 43.11 & 43.11 \\
\hline 16 & 125.59 & 119.59 & 35.09 & 32.18 \\
\hline 17 & 133.71 & 119.32 & 37.49 & 31.20 \\
\hline 18 & 134.44 & 120.56 & 33.77 & 27.76 \\
\hline 19 & 129.16 & 117.72 & 40.75 & 35.56 \\
\hline 20 & 122.78 & 118.99 & 50.28 & 50.27 \\
\hline Mean & 126.85 & 120.19 & 38.06 & 33.93 \\
\hline
\end{tabular}

(GA: gonial angle, MPA: mandibular plane angle, 2D: two-dimensional, 3D CT: three-dimensional computed tomography)

Suseok Oh et al: A comparative study between data obtained from conventional lateral cephalometry and reconstructed three-dimensional computed tomography images. J Korean Assoc Oral Maxillofac Surg 2014

ally used with lateral cephalometry. Panorama radiography is the most familiar technique to most dentists and orthodontists, and it is the representative examination for dental diagnosis and treatment planning. This type of imaging enables dentists to detect cysts and tumors, supernumerary teeth, missing teeth, and bony deformities and to evaluate the asymmetry of the lower jawbone ${ }^{19,20}$. Updegrave ${ }^{21}$, however, reported that an incorrect treatment plan may results from serious asymmetry of the mandible ramus and condyle and coronoid processes on panorama radiography. Other authors have reported difficulty in analyzing the face with panorama radiography ${ }^{22}$. Posteroanterior cephalometry has the same problem in that precise perception is difficult because of many landmark overlaps ${ }^{13}$. As such, there are limitations to the evaluation of $3 \mathrm{D}$ structures on $2 \mathrm{D}$ radiographic images.

However, no overlapping landmarks are produced in 3D imaging, minimizing the confusion of left- and right-side structures. Therefore, with 3D CT imaging, clinicians can diagnose all patients accurately including those with facial asymmetry, easily identify the shapes of craniofacial structures, and recognize the shapes of bones and soft tissues from various angles. According to previous studies measuring length on volume-rendered 3D CT images and in cadavers ${ }^{23}$, the difference in length between two groups was minimal, confirming the accuracy of 3D-CT images. Hildebolt et al. ${ }^{24}$ also reported accuracy of measurement on 3D CT.

In addition, 3D lateral cephalometry analysis abstracted from 3D CT was introduced, and its accuracy has been reported. van Vlijmen et al. ${ }^{1}$ performed comparative analysis on various angles and distances between 3D lateral cephalometry and conventional lateral cephalometry and reported that there was no statistically significant difference in many parts.

It is true that lateral cephalometry analysis has been the major method to evaluate facial types for tens of years ${ }^{15,25} .3 \mathrm{D}$ CT analysis, a very useful method in diagnosis, uses the same landmarks as conventional lateral cephalometry ${ }^{15}$. However, the concordance between the measurements of lateral cephalometry and 3D CT has not been sufficiently studied. Using the relationship and patterns of measurements acquired from two analysis methods, as well as their concordance, 3D CT values can be determined from the values of lateral cephalometry analysis. Thus, accumulated lateral cephalometry values can be converted to 3D. However, few cases have performed a clear comparison between the two methods.

The UOPA abstracted from the FH plane defined in 3D CT and that from lateral cephalometry were found to be very similar.(Table 2) Similarly, the four 3D FH planes did not show differences in concordance with those of $2 \mathrm{D}$ analysis. In such cases, it is desirable to choose landmarks with higher 
representation and reliability. In a study by $\mathrm{Yu}$ et al. ${ }^{26}$, two observers marked lateral cephalometry landmarks three times and calculated the concordance with respect to the $\mathrm{X}$-axis and $y$-axis. In this experiment, Po showed the lowest concordance among 17 landmarks on the $\mathrm{x}$-axis, while Or did not show a significant difference in concordance among observers or among trials of the same observer. Additionally, when defining Po on 3D CT, a wide curve is produced, making repetition and reproduction difficult.(Fig. 1) Based on these results, it is reasonable to define the $\mathrm{FH}$ plane in $3 \mathrm{D}$ using two Or and one Po values.

According to the results of this study, GA measured on 2D is larger than that measured on 3D in all patients.(Table 4) There are three possible explanations. First, the difference may be because of error in menton (Me). When marking Me on 3D CT (Fig. 2B) and observing the resulting 3D lateral cephalometry, Me marked on the superior position that $\mathrm{Me}$ on conventional lateral cephalometry.(Fig. 2C) This occurs because anatomic structures near the Me are extended in the lower direction on 3D CT.(Fig. 2A) In patients with such structures, the $3 \mathrm{D}$ values may be different from those on $2 \mathrm{D}$ even when Me is accurately marked on 3D CT. Additionally, when Me is marked at the front of skull, it may be marked in a little superior position (Fig. 2D), and it may lead to erroneous underestimation of GA and MPA. Thus, when Me is marked on $3 \mathrm{D} \mathrm{CT}$, it is recommended to approach from the bottom of the lower jawbone.

Second, articulare (Ar) may have larger $\mathrm{x}$-axis error on lateral cephalometry compared with 3D CT. Trpkova et al. ${ }^{7}$ confirmed through meta-analysis that $\mathrm{Ar}$ and basion showed
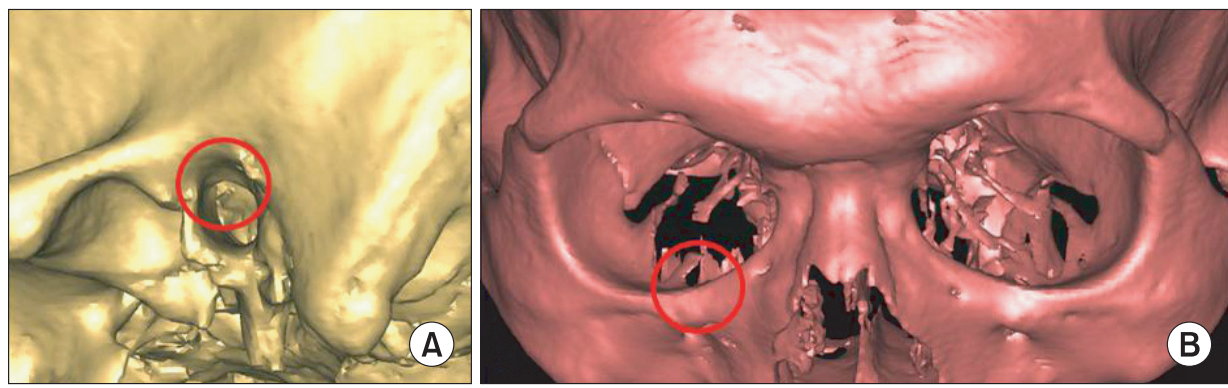

Fig. 1. A. Large, round-shaped bony edge around the porion. B. Sharp edge around the orbitale.

Suseok Oh et al: A comparative study between data obtained from conventional lateral cephalometry and reconstructed three-dimensional computed tomography images. J Korean Assoc Oral Maxillofac Surg 2014
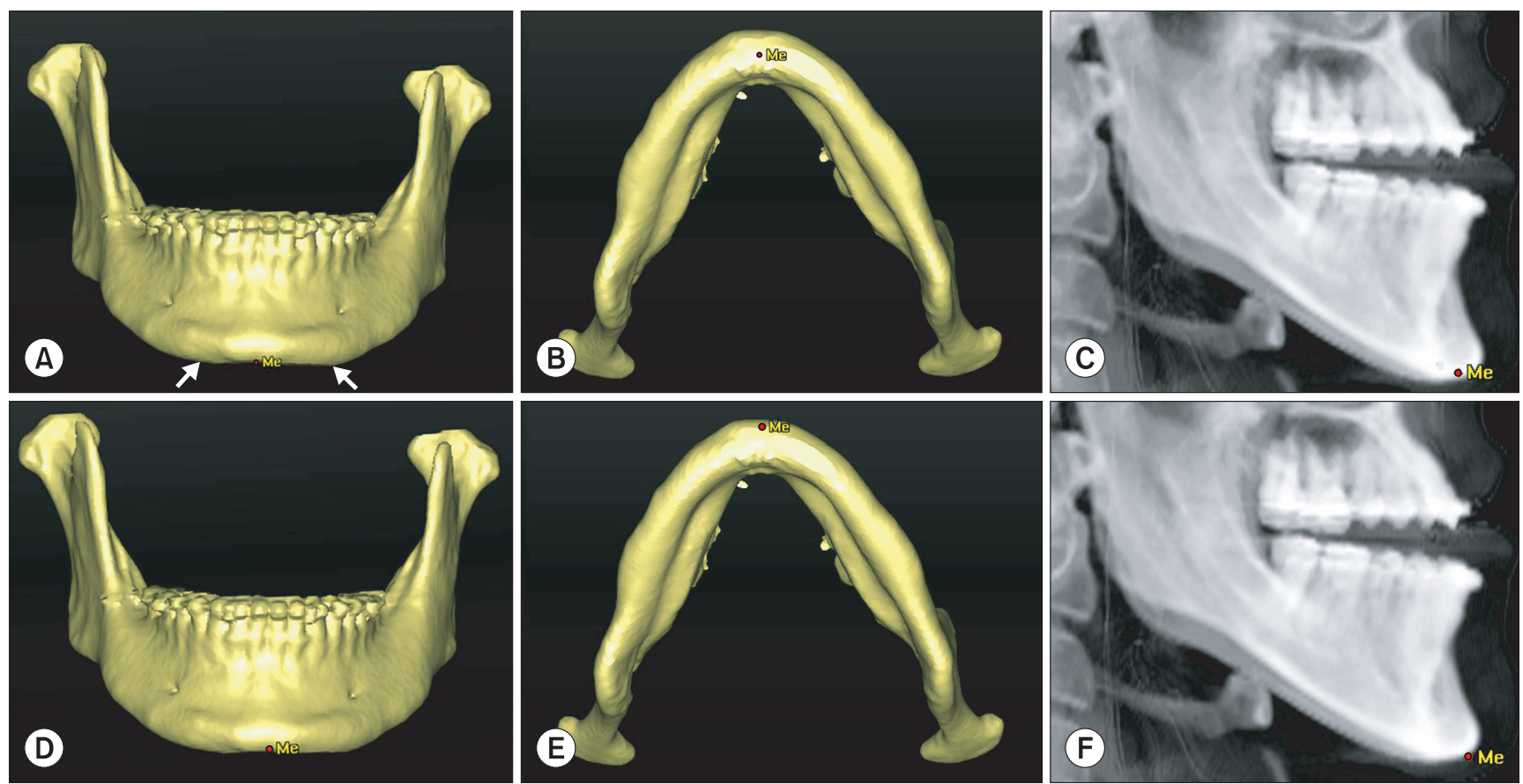

Fig. 2. A. Downward extended adjacent structure on both sides (white arrows). B. Position of the menton (Me) in the inferior-superior direction. C. Position of the Me on three-dimensional (3D) cephalometry. D. Me positioned in the anterior-posterior view. E. Me positioned in the anterior view. F. Position of the Me on 3D cephalometry.

Suseok Oh et al: A comparative study between data obtained from conventional lateral cephalometry and reconstructed three-dimensional computed tomography images. J Korean Assoc Oral Maxillofac Surg 2014 
the largest $\mathrm{x}$-axis errors on lateral cephalometry. The possibility of lower accuracy of GA cannot be excluded because of lower accuracy of the reference points.

Third, Ar defined in 3D is different from the definition in 2D. Ar on lateral cephalometry is defined as the point of overlap between the skull base and posterior surface of the lower jawbone condyle, while Ar in the present study is defined as the most posterior point of the mandibular condyle. Thus, it is difficult to assert that Ar in this study is absolutely concordant with Ar on 2D. However, it is impossible to define $\mathrm{Ar}$ in $3 \mathrm{D}$ using the same definition of that in $2 \mathrm{D}$. Therefore, if Ar is defined using the method of this study, GA in $2 \mathrm{D}$ is slightly larger than that in $3 \mathrm{D}$ CT.

MPA, just like GA, was larger in 2D than in 3D in all patients. This can be explained by the abovementioned problems associated with Me.

The angles showing low concordance were palatal angle, U1OPA, and U1FHPA.(Table 3) An angle is made by two lines; if the lines are short, the measurement is more difficult, and the risk of error increases, which lowers the probability of repetition and reproduction and consequently the probability of concordance. The straight palatal line and U1 line used to define the aforementioned three angles had short distances, which resulted in their lower concordance values. On the other hand, MPA, UOPA, SNA, and SNB were formed by two long lines and so showed higher concordance.

\section{Conclusion}

For measurement of angles made up of two long lines, the concordance between two methods is high. In this study, we showed high concordance for MPA, UOPA, SNA, and SNB. When Me was included in the analysis, it was marked in a different location from that of $2 \mathrm{D}$ analysis because of the nature of the measurement points. Additionally, the $\mathrm{FH}$ plane on 3D CT showed concordance with the FH plane on lateral cephalometry, indicating no difference in concordance no matter what is selected among four FH planes defined on 3D CT. However, it is desirable to define the FH plane on 3D CT with two Or and one Po values considering the reproduction of Or itself.

\section{Conflict of Interest}

No potential conflict of interest relevant to this article was reported.

\section{References}

1. van Vlijmen OJ, Bergé SJ, Swennen GR, Bronkhorst EM, Katsaros C, Kuijpers-Jagtman AM. Comparison of cephalometric radiographs obtained from cone-beam computed tomography scans and conventional radiographs. J Oral Maxillofac Surg 2009;67:92-7.

2. Athanasiou AE. Orthodontic cephalometry. London: MosbyWolfe; 1997.

3. Adams GL, Gansky SA, Miller AJ, Harrell WE Jr, Hatcher DC. Comparison between traditional 2-dimensional cephalometry and a 3-dimensional approach on human dry skulls. Am J Orthod Dentofacial Orthop 2004;126:397-409.

4. Miller PA, Savara BS, Singh IJ. Analysis of errors in cephalometric measurement of three-dimensional distances on the maxilla. Angle Orthod 1966;36:169-75.

5. Gravely JF, Benzies PM. The clinical significance of tracing error in cephalometry. Br J Orthod 1974;1:95-101.

6. Midtgård J, Björk G, Linder-Aronson S. Reproducibility of cephalometric landmarks and errors of measurements of cephalometric cranial distances. Angle Orthod 1974;44:56-61.

7. Trpkova B, Major P, Prasad N, Nebbe B. Cephalometric landmarks identification and reproducibility: a meta analysis. Am J Orthod Dentofacial Orthop 1997;112:165-70.

8. Wylie GA, Fish LC, Epker BN. Cephalometrics: a comparison of five analyses currently used in the diagnosis of dentofacial deformities. Int J Adult Orthodon Orthognath Surg 1987;2:15-36.

9. Magalhaes AE, Stella JP, Epker BN. Facial anthropometrics versus cephalometry as predictors for surgical treatment in patients with class III dentofacial deformities. Int J Adult Orthodon Orthognath Surg 1995;10:295-302.

10. Swennen GR, Schutyser F. Three-dimensional virtual approach to diagnosis and treatment planning of maxillo-facial deformity. In: Bell W, ed. Distraction osteogenesis of the facial skeleton. Hamilton, ON: BC Decker Inc; 2007:55.

11. Maki K, Okano T, Morohashi T, Yamada S, Shibaski Y. The application of three-dimensional quantitative computed tomography to the maxillofacial skeleton. Dentomaxillofac Radiol 1997;26:3944.

12. Vannier MW, Marsh JL, Warren JO. Three dimensional CT reconstruction images for craniofacial surgical planning and evaluation. Radiology 1984;150:179-84.

13. Trpkova B, Prasad NG, Lam EW, Raboud D, Glover KE, Major PW. Assessment of facial asymmetries from posteroanterior cephalograms: validity of reference lines. Am J Orthod Dentofacial Orthop 2003;123:512-20.

14. Wong RW, Chau AC, Hägg U. 3D CBCT McNamara's cephalometric analysis in an adult southern Chinese population. Int J Oral Maxillofac Surg 2011;40:920-5.

15. Maeda M, Katsumata A, Ariji Y, Muramatsu A, Yoshida K, Goto S, et al. 3D-CT evaluation of facial asymmetry in patients with maxillofacial deformities. Oral Surg Oral Med Oral Pathol Oral Radiol Endod 2006; 102:382-90.

16. Chebib FS, Chamma AM. Indices of craniofacial asymmetry. Angle Orthod 1981;51:214-26.

17. Epker BN, Fish LC, Stella JP. Dentofacial deformities: integrated orthodontic and surgical correction. St Louis: CV Mosby; 1998:2933.

18. Raustia AM, Salonen MA. Gonial angles and condylar and ramus height of the mandible in complete denture wearers--a panoramic radiograph study. J Oral Rehabil 1997;24:512-6.

19. Piedra I. The Levandoski panoramic analysis in the diagnosis of facial and dental asymmetries. J Clin Pediatr Dent 1995;20:15-21.

20. Mattila M, Könönen M, Mattila K. Vertical asymmetry of the mandibular ramus and condylar heights measured with a new method from dental panoramic radiographs in patients with psoriatic arthritis. J Oral Rehabil 1995;22:741-5. 
21. Updegrave WJ. Visualizing the mandibular ramus in panoramic radiography. Oral Surg Oral Med Oral Pathol 1971;31:422-9.

22. Yeo DK, Freer TJ, Brockhurst PJ. Distortions in panoramic radiographs. Aust Orthod J 2002;18:92-8.

23. Cavalcanti MG, Haller JW, Vannier MW. Three-dimensional computed tomography landmark measurement in craniofacial surgical planning: experimental validation in vitro. J Oral Maxillofac Surg 1999;57:690-4.
24. Hildebolt CF, Vannier MW, Knapp RH. Validation study of skull three-dimensional computerized tomography measurements. Am J Phys Anthropol 1990;82:283-94.

25. Mulick JF. Clinical use of the frontal headfilm. Angle Orthod 1965;35:299-304.

26. Yu SH, Nahm DS, Baek SH. Reliability of landmark identification on monitor-displayed lateral cephalometric images. Am J Orthod Dentofacial Orthop 2008;133:790.e1-6; discussion e1. 